\title{
Cultural Integration and the Accessibility of Gaming
}

\author{
Michael James Heron ${ }^{1}$ (D)
}

Published online: 3 November 2016

(C) Springer Science+Business Media New York 2016

It is easy to see that video games are becoming increasingly more inventive, innovative and immersive. We see, every year, a staggeringly diverse array of titles. These are produced by organisations as varied as transnational corporations, midsized indies, and bedroom coders. Twenty Sixteen alone has seen the release of games as varied as No Man's Sky; Deus Ex: Mankind Divided; the Telltale Games Batman Series; Overwatch; Duskers; and the Duelyst. In the modern gaming cornucopia, players are truly spoiled for choice. There is something there for everyone, and everyone can find something.

Most of the time.

The reality of the matter is that the provision of popular gaming entertainment exists in a two-tier system. There are those that can enjoy the products of a video game industry at the height of its creativity, and those that are excluded from full participation. This is not as a result of malice or intentionally exclusionary design, but as a consequence of often inadvertent inaccessibility. Many games remain, even now, obstinately inaccessible in a societal context that stresses the importance of inclusion. This is an issue that plagues video games specifically, but also has its direct parallel with the accessibility of other, more tactile products such as table-top titles, and the overlap between apps and designer board-games.

In times of increased financial pressures, intense competition, and often highly expensive development cycles it is surprising that this should be the case. There is a substantial, largely untapped marketplace, consisting of gamers with physical, cognitive, auditory or visual impairments. Accessibility makes sound business sense.

I have had this conversation with many people, and some-even those most affected by issues of accessibility_consider it to be a matter of limited priority.

Michael James Heron

m.j.heron1@rgu.ac.uk

1 Robert Gordon University, Aberdeen AB10 7QB, Scotland, UK 
'There's so much inaccessibility in the stuff I need for me to worry about the inaccessibility in the stuff I want'. Equitable access to the cultural products of society is seen by some as of secondary importance-nice to have, but deserving of only marginal attention. There are, people argue, no real downsides to people not being able to play the games they want to play.

That is a stance with which I strongly disagree.

Arguments rage over whether games are art, or whether they are too violent, or not violent enough, whether they are inclusive, whether they should be inclusive, and whether games can rival movies or books for emotional impact. These are interesting, vibrant, and meaningful discussions that are far from being resolved. The hidden debate at the core of all of these topics is not 'do games matter', but 'to what extent do they matter?'

For me, games exist not just as entertainment products but powerful tools of social integration (Kaye and Bryce 2012). When we can share our experiences of participation with the people around us, we build cultural capital. That cultural capital permits us to feel included in the wider conversation of society. Facilitated by the internet, consumption of entertainment is no longer a strictly passive activity-we do not simply read a book and then put it down. We talk about it, how it made us feel, what it made us think. When we watch Game of Thrones, we are compelled at the end to hop on social media to enthuse (or rage) about the course the narrative took. So too it is with games-we want to be able to talk with the people around us about the experiences we have had within their worlds. This is an intensely creative act-one of interpretation that constructs collaborative critical perspectives that reinforce the work itself. These critical perspectives help shape and inform the narratives in our own lives, and helps us build connections around a set of shared cultural experiences. They provide insight. They connect us together. Nowhere is this truer than in games, where our active participation is a fundamental act of story-telling. We laugh, and joke, and tease about the mistakes we and others make. Our most hilarious failures are anecdotes. Our successes are personal mythology. In a very real sense, games contribute to the bonds that tie us together. Play is an inherently shared experience - the young of all species play. Games are a pure expression of that-whether it's playing chess, Sudoko, or Grand Theft Autowe all understand the intrinsic social value of games.

Inaccessibility then robs individuals of their ability to contribute to the wider context of society. It mutes their voices, since they cannot engage with discussion as freely or as easily. And in turn, representation of important, marginalised voices becomes less common in works of fiction. As a result only rarely can people with disabilities see themselves reflected in works of popular impact, and even then rarely with the necessary sensitivity or surety of touch to be anything other than insulting. If you cannot participate in the construction of popular culture even through consumption you can't help shape its eventually direction.

This is disempowering. It is alienating. It is exclusionary. And it doesn't soften any of this much to know that it is often accidental and unintentional (Heron 2012).

Many of the tools that are used to build gaming experiences are contributors to this problem-focusing on the workflow of creation, they rarely consider the day to day realities of complex, multi-spectrum interaction compensation. They abstract 
away key APIs, or implement their own systems with little reference to the accessibility standards that exist in the wider software ecosystem. Ensuring accessibility within games, as it currently stands, is a task that must be actively pursued by motivated individuals and often through the wrestling of reluctant and recalcitrant game engines.

There are resources out there to assist with this, and many of them are very good. For video games, there are the Game Accessibility Guidelines (http:// gameaccessibilityguidelines.com/), and the Includification framework (http:// www.includification.com/). For table-top games, my own project Meeple Like Us (http://meeplelikeus.co.uk/) is currently working on mapping out the accessibility landscape. While guidelines by themselves are sometimes unhelpful, there is a motivated community of scholars, gamers, developers and designers that are desperate to help. All that you need to do is say 'I want to maximise the accessibility of my game', and you'll have more genuinely useful advice than you could ever wish for.

There is though cause to be positive - the last year has been a good one for the game accessibility agenda.

Naughty Dog gained much popular support for the accessibility work they put into Uncharted 4, showing that this is an area where attention can yield real marketplace results. Overwatch too shines in terms of the careful consideration it has invested into ensuring an accessible gaming experience. Microsoft made accessibility one of its key deliverables as part of its E3 announcements. Game accessibility is becoming a mainstream issue, and it is going to change the landscape of production and design dramatically for the better if we can all just get behind it.

Accessible interfaces are not a niche consideration. Accessible design is not a luxury. Accessible interfaces are better for everyone-there is little difference between someone with extraordinary interaction requirements, and someone with ordinary requirements in an extraordinary context (Newell and Gregor 2000). The accessibility support that permits someone with hand tremor to play a mobile phone game is the same support that benefits me when playing on a juddering bus. The close captioning that permits the hearing impaired to play their favourite franchises is the same I can use to play whilst watching a movie or listening to music. More than this, the demographic of dedicated gamers is aging all the time. We are all getting older with each passing second. With age comes a broad spectrum of accumulated impairment in all categories. We all become more extraordinary as time goes by. I don't know about you, but during my retirement I want to be able to play all the games for which I have never been able to find the time. If nothing else, our own inherent hedonistic selfishness should mandate a greater push for game accessibility. When we say 'So everyone can play', even those for whom accessibility is not currently an issue must have their future selves in mind.

I end here with a short quote from Silas Humphreys, a disabled gamer writing on the Game Accessibility Guidelines page. It really sums up everything that needs to be said.

This should be required reading. Because we exist, and we want to buy your games. 


\section{References}

Heron, M. (2012). Inaccessible through oversight: The need for inclusive game design. Computer Games Journal, 1(1), 29-38.

Kaye, L. K., \& Bryce, J. (2012). Putting the fun factor into gaming: The influence of social contexts on the experiences of playing videogames. International Journal of Internet Science, 7(1), 24-38.

Newell, A. F., \& Gregor, P. (2000). User sensitive inclusive design-In search of a new paradigm. In Proceedings on the 2000 conference on universal usability (pp. 39-44) ACM. 